\title{
Migrânea é comórbida ao transtorno do déficit de atenção e hiperatividade na infância: um estudo populacional nacional
}

\author{
Migraine, tension-type headache, and attention-deficit/Hyperactivity disorder in childhood: \\ A population-based study
}

\author{
Marco Antônio Arruda ${ }^{1}$, Renato Arruda ${ }^{2}$, Vincenzo Guidetti ${ }^{3}$, Marcelo Eduardo Bigal ${ }^{4}$ \\ ${ }^{1}$ IG - Instituto Glia, Ribeirão Preto, SP; \\ ${ }^{2}$ FCM/Unicamp - Faculdade de Ciências Médicas da Unicamp - Campinas, SP \\ ${ }^{3}$ La Sapienza - Universidade de Roma "La Sapienza" - Roma, Itália \\ ${ }^{4} \mathrm{AECM}$ - Albert Einstein College of Medicine, Estados Unidos
}

Arruda MA, Arruda R, Guidetti V, Bigal ME. Migrânea é comórbida ao transtorno do déficit de atenção e hiperatividade na infância: um estudo populacional nacional. Headache Medicine. 2017;8(1):11-5

\section{INTRODUÇÃO}

A migrânea e o Transtorno do Déficit de Atenção e Hiperatividade (TDAH) apresentam uma alta prevalência, acometendo, respectivamente, em torno de $5 \%$ e $10 \%$ da população infantil mundia|(1,2) (Figura 1), provocando um impacto clinicamente significativo e de grau variado na qualidade de vida, ${ }^{(3)}$ bem-estar psicológico, ${ }^{(4)}$ desempenho escolar ${ }^{(5)}$ e ajuste psicossocial da criança. ${ }^{(6)}$ Ambas as condições se encontram no grupo das doenças crônicas não comunicáveis, hoje consideradas pela Organização Mundial de Saúde como as principais causas de morbidade na infância, exigindo programas específicos de saúde pública.

A possível comorbidade entre a migrânea e o TDAH é cogitada na literatura apenas por estudos clínicos, carecendo de evidências advindas de estudos populacionais representativos, não influenciados por vieses de seleção.

No presente estudo, apresentamos resultados obtidos em uma amostra populacional em que as crianças foram randomicamente selecionadas em suas escolas e seus pais e professores foram diretamente entrevistados (Projeto Atenção Brasil), a fim de investigar se o TDAH e/ ou os sintomas de hiperatividade-impulsividade e desatenção são comórbidos à migrânea e/ou à cefaleia do tipo tensional (CTT).

\section{MÉTODO}

Projeto Atenção Brasil foi desenhado com o objetivo de estabelecer coortes para estudar aspectos do desenvolvimento infantil. Em sua fase nacional, contou com a participação voluntária de professores de todo o Brasil que fazem parte da Comunidade Aprender Criança, uma comunidade acadêmica, virtual e sem fins lucrativos, criada em 2006 com o objetivo de desenvolver a interface entre as Neurociências e a Educação. A partir de um convite aberto a todos educadores da referida comunidade, um planejamento amostral identificou 124 professores, que, em seguida, completaram um treinamento on-line de 4 horas conduzido por um de nós (MAA) com orientações sobre o processo de seleção da amostra e o preenchimento dos questionários.

Os professores completaram a versão brasileira validada da escala MTA-SNAP-IV7 para identificação de sintomas do TDAH, além de um módulo referente ao desempenho escolar, para cada um dos seus alunos incluídos na amostra. Em seguida, os pais foram entrevistados pelos professores através de um questionário padronizado com 102 perguntas que avaliam características sociodemográficas, antecedentes pessoais, cefaleia e saúde mental. $\bigcirc$ módulo de cefaleia, composto por 14 questões que avaliam aspectos distintivos das cefaleias de acor- 


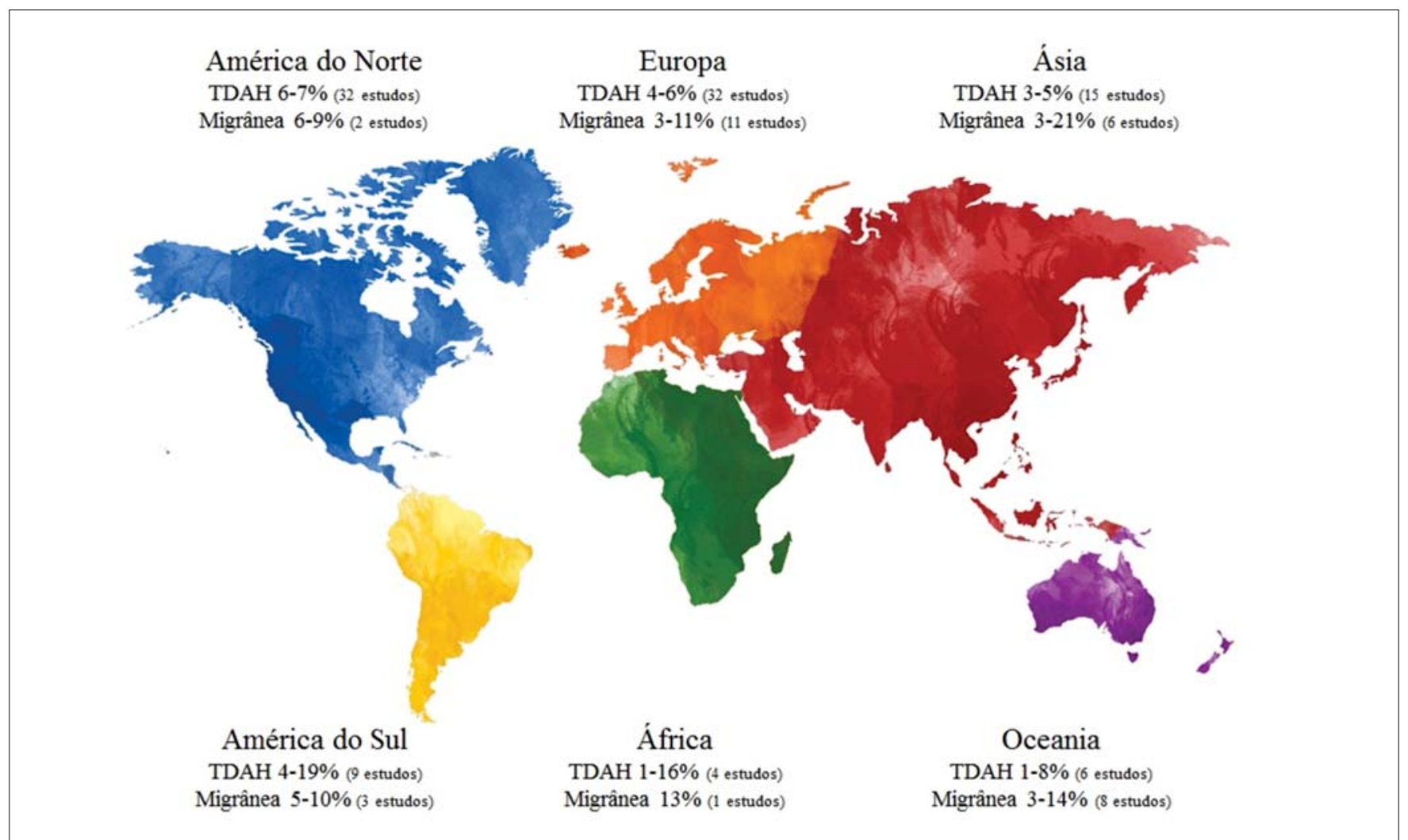

Figura 1. Mapa-mundi da prevalência de TDAH e migrânea em crianças e adolescentes ${ }^{(1,2)}$

do com a segunda edição da Classificação Internacional das Cefaleias (ICHD-2), foi validado em numerosos estudos brasileiros com adultos e crianças. O estado de saúde mental da criança foi avaliado pela versão brasileira validada do Questionário de Capacidades e Dificuldades (SDQ)8, além da escala MTA-SNAP-IV. OSDQ identifica hiperatividade, desatenção, sintomas emocionais, problemas de relacionamento com colegas e problemas de conduta. O suplemento de impacto do SDQ foi aplicado para avaliar a presença de comprometimento clinicamente significativo dos sintomas do TDAH nos vários contextos de vida da criança, requerido pelos critérios operacionais de diagnóstico do DSM-IV.

Para caracterização da amostra foi utilizada estatística descritiva e as taxas de prevalência bruta e ajustada foram obtidas utilizando modelo de regressão binária. Foram construídos modelos para análise multivariada por regressão logística que avaliam a presença de TDAH em função do diagnóstico da cefaleia, idade, sexo, raça, renda familiar, características clínicas da cefaleia, bem como a presença de abuso de analgésicos. $\bigcirc$ nível de significância adotado foi de 5\%. A análise estatística foi realizada com o auxílio do programa SPSS 15.0 para Windows (SPSS Inc. Chicago, IL).

\section{RESULTADOS}

Das 8.599 crianças da amostra-alvo, um consentimento informado dos pais foi obtido em 6.445 (75\%) e questionários com informações completas e analisáveis em 5.671 (65,9\%), a amostra final. A idade variou de 5 a 12 anos, sendo $50,7 \%$ delas do sexo masculino, oriundas de 87 cidades de 18 estados brasileiros, representando as cinco regiões nacionais (Tabela 1).

A prevalência de TDAH foi de 3,9\% (6,0\% em meninos e 1,8\% em meninas), migrânea episódica 9,4\% e CTT episódica em 12,6\% da amostra. Em comparação às crianças sem cefaleia, a prevalência de TDAH foi significativamente maior em crianças com migrânea em geral ( $6 \%$ vs. $2,1 \%$, RR 2,9, IC 95\% 1,9-4,5), migrânea episódica (6,8\% vs. 2,1\%, RR 3,3 IC 95\% 2,0-5,3), provável migrânea $(5,3 \%$ vs. 2,1\%, RR 2,5; IC95\% 1,6$3,4)$, migrânea crônica $(17,1 \%$ vs. $2,1 \%$, RR 8,2; IC 95\% 3,8-17,9), CTT em geral (3,6\% vs. 2,1\%, RR 1,7; IC95\% 1, 1-2,7) e provável CTT (3,7\% vs. 2,1\%, RR 1,8; IC95\% 1,2-2,8). Em relação aos sintomas de hiperatividade e impulsividade, a prevalência foi significativamente maior em crianças com migrânea em geral, migrânea episódica e provável migrânea em compara- 
Tabela 1 - Amostra-alvo, respondentes e amostra final de acordo com as características demográficas

\begin{tabular}{|c|c|c|c|c|c|c|}
\hline & \multirow{3}{*}{$\frac{\text { Amostra-alvo }}{n}$} & \multirow{2}{*}{\multicolumn{2}{|c|}{ Respondentes }} & \multirow{3}{*}{$\begin{array}{c}\text { Amostra final } \\
\mathrm{n}\end{array}$} & \multicolumn{2}{|c|}{ Taxa de participação } \\
\hline & & & & & $\begin{array}{c}\text { Relativo à } \\
\text { amostra-alvo }\end{array}$ & $\begin{array}{l}\text { Relativo aos } \\
\text { respondentes }\end{array}$ \\
\hline & & $\mathrm{n}$ & $\%$ & & $\%$ & $\%$ \\
\hline \multicolumn{7}{|l|}{ Grupo etário } \\
\hline 5 a 8 & 4.801 & 3.657 & 76,2 & 3.111 & 64,8 & 85,1 \\
\hline 9 a 12 & 3.798 & 2.788 & 73,4 & 2.560 & 67,4 & 91,8 \\
\hline \multicolumn{7}{|l|}{ Gênero } \\
\hline Feminino & 4.259 & 3.186 & 74,8 & 2.794 & 65,6 & 87,7 \\
\hline Masculino & 4.340 & 3.259 & 75,1 & 2.877 & 66,3 & 88,3 \\
\hline \multicolumn{7}{|l|}{ Ano escolar } \\
\hline 10. & 1.538 & 1.160 & 75,4 & 1.044 & 67,9 & 90,0 \\
\hline 20. & 2.384 & 1.884 & 79,0 & 1.674 & 70,2 & 88,9 \\
\hline 3o. & 1.716 & 1.252 & 73,0 & 1.105 & 64,4 & 88,3 \\
\hline 40. & 2.053 & 1.490 & 72,6 & 1.272 & 62,0 & 85,4 \\
\hline 50. & 908 & 659 & 72,6 & 576 & 63,4 & 87,4 \\
\hline \multicolumn{7}{|l|}{ Raça } \\
\hline Branca & 5.856 & 4.198 & 71,7 & 3.769 & 64,4 & 89,8 \\
\hline Não branca & 2.743 & 1.964 & 71,6 & 1.672 & 61,0 & 85,1 \\
\hline Não respondentes & & 283 & & 230 & & 81,3 \\
\hline \multicolumn{7}{|l|}{ Classe econômica } \\
\hline$A, B$ & 3.034 & 2.332 & 76,9 & 2.069 & 68,2 & 88,7 \\
\hline $\mathrm{C}$ & 4.347 & 3.234 & 74,4 & 2.856 & 65,7 & 88,3 \\
\hline$D, E$ & 1.218 & 879 & 72,2 & 746 & 61,2 & 84,9 \\
\hline \multicolumn{7}{|l|}{ Região } \\
\hline Norte & 278 & 172 & 61,9 & 134 & 48,2 & 77,9 \\
\hline Nordeste & 1.245 & 912 & 73,3 & 712 & 57,2 & 78,1 \\
\hline Centro-Oeste & 405 & 298 & 73,6 & 188 & 46,4 & 63,1 \\
\hline Sudeste & 3.778 & 2.837 & 75,1 & 2.542 & 67,3 & 89,6 \\
\hline Sul & 2.893 & 2.226 & 76,9 & 2.095 & 72,4 & 94,1 \\
\hline \multicolumn{7}{|c|}{ Densidade populacional } \\
\hline$<100.000$ & 3.245 & 2.567 & 79,1 & 2.220 & 68,4 & 86,5 \\
\hline $100.000-500.000$ & 3.546 & 2.589 & 73,0 & 2.365 & 66,7 & 91,3 \\
\hline$>500.000$ & 1.808 & 1.289 & 71,3 & 1.086 & 60,1 & 84,3 \\
\hline Total & 8.599 & 6.445 & 75,0 & 5.671 & 65,9 & 88,0 \\
\hline
\end{tabular}

ção com as crianças sem cefaleia $(p<0,04)$. Em comparação com as crianças sem cefaleia, a prevalência de sintomas de desatenção foi significativamente maior em crianças com migrânea em geral, migrânea episódica, provável migrânea, migrânea crônica, CTT em geral e provável CTT ( $p<0,03)$ (Tabela 2).

De acordo com a análise multivariada, em crianças com migrânea em geral, migrânea episódica, provável migrânea e migrânea crônica, o diagnóstico de TDAH foi significativa e independentemente influenciado pelo sexo masculino $(p<0,0001)$, alta frequência $(p<0,04)$ e forte intensidade $(p<0,001)$ da cefaleia, presença de náuseas $(p<0.0001)$ e fonofobia $(p<0,024)$ nas crises de cefaleia, presença de problemas de conduta $(p<0,008)$, um escore total anormal do SDQ ( $p<0,0001)$ e um desempenho escolar abaixo da média ( $p<0,0001)$ (Tabela 3). 
Tabela 2 - Prevalência do TDAH e dos sintomas de hiperatividade, impulsividade e desatenção em função do diagnóstico da cefaleia

\begin{tabular}{|c|c|c|c|c|c|c|c|c|c|c|}
\hline & \multirow{2}{*}{$\begin{array}{c}\begin{array}{c}\text { Amostra } \\
\text { final }\end{array} \\
n(\%)\end{array}$} & \multicolumn{3}{|c|}{ TDAH (DSM-IV) } & \multicolumn{3}{|c|}{$\begin{array}{c}\text { Sintomas de Hiperatividade } \\
\text { e Impulsividade }\end{array}$} & \multicolumn{3}{|c|}{ Sintomas de Desatenção } \\
\hline & & $\mathrm{n}(\%)$ & $\begin{array}{c}\mathrm{RR} \\
(95 \% \mathrm{IC}) \\
\end{array}$ & $\mathrm{p}$ & $\mathrm{n}(\%)$ & $\begin{array}{c}\mathrm{RR} \\
(95 \% \mathrm{IC})\end{array}$ & $\mathrm{p}$ & $\mathrm{n}(\%)$ & $\begin{array}{c}\mathrm{RR} \\
(95 \% \mathrm{IC})\end{array}$ & $\mathrm{p}$ \\
\hline Sem cefaleia & $1.168(20,6)$ & $26(2,1)$ & Referência & & $37(3,0)$ & Referência & & $43(3,4)$ & Referência & \\
\hline Migrânea em geral & $1.565(27,6)$ & $107(6,0)$ & $2.9(1.9-4.5)$ & $<0,001$ & $85(4,8)$ & $1.6(1.1-2.4)$ & 0,01 & $124(7,0)$ & $2.0(1.5-2.9)$ & $<0,001$ \\
\hline Migrânea Episódica & $533(9,4)$ & $40(6,8)$ & $3.3(2.0-5.3)$ & $<0,001$ & $30(5,1)$ & $1.7(1.1-2.8)$ & 0,03 & $46(7,8)$ & $2.3(1.5-3.4)$ & $<0,001$ \\
\hline Provável Migrânea & $998(17,6)$ & $60(5,3)$ & $2.5(1.6-3.4)$ & $<0,001$ & $53(4,7)$ & $1.6(1.0-2.4)$ & 0,04 & $71(6,2)$ & $1.8(1.3-2.6)$ & $<0,001$ \\
\hline Migrânea Crônica & $34(0,6)$ & $7(17,1)$ & $8.2(3.8-17.9)$ & $<0,001$ & $2(4,9)$ & $1.7(0.4-6.6)$ & 0,35 & $7(17,1)$ & $5.0(2.4-10.4)$ & $<0,001$ \\
\hline CTT em geral & $2.938(51,8)$ & $100(3,6)$ & $1.7(1.1-2.7)$ & 0,01 & $103(3,7)$ & $1.3(0.9-1.8)$ & 0,26 & $141(5,1)$ & $1.5(1.1-2.1)$ & 0,02 \\
\hline CTT Episódica & $714(12,6)$ & $26(3,3)$ & $1.6(0.9-2.7)$ & 0,12 & $23(2,9)$ & $1.0(0.6-1.6)$ & 0,95 & $40(5,1)$ & $1.5(1.0-2.2)$ & 0,09 \\
\hline Provável CTT & $2.223(39,2)$ & $74(3,7)$ & $1.8(1.2-2.8)$ & 0,01 & $80(4,0)$ & $1.4(0.9-2.0)$ & 0,13 & $101(5,1)$ & $1.5(1.1-2.1)$ & 0,03 \\
\hline CTT Crônica & $1(0,0)$ & 0 & 0 & 1,00 & 0 & 0 & 1,00 & 0 & 0 & 1,00 \\
\hline
\end{tabular}

TDAH: Transtorno do déficit de atenção e hiperatividade; Migrânea episódica: migrânea sem e com aura

CTT: Cefaleia do tipo tensional

Tabela 3 - Análise multivariada dos determinantes no diagnóstico do TDAH em crianças com migrânea em geral

\begin{tabular}{lccc}
\hline & Wald Chi-Square & $p$ & $\begin{array}{c}\text { Odds Ratio } \\
(95 \% \text { IC })\end{array}$ \\
\hline Sexo masculino & 19,44 & $<0,0001$ & $3,62(2,04-6,41)$ \\
CEFI vs. CEBF & 6,34 & 0,012 & $2,44(1,22-4,88)$ \\
CEAF vs. CEBF & 5,23 & 0,021 & $3.12(1,18-8,23)$ \\
CCD vs. CEAF & 4,21 & 0,040 & $3,07(1,05-8,94)$ \\
Intensidade moderada/forte vs.fraca & 10,50 & 0,001 & $3,84(1,70-8,68)$ \\
Náusea & 17,28 & $<0,0001$ & $3,23(1,86-5,61)$ \\
Fonofobia & 5,07 & 0,024 & $1,86(1,08-3,18)$ \\
Problemas de conduta & 7,00 & 0,008 & $2,58(1,28-5,19)$ \\
Escore total anormal (SDQ) & 17,13 & $<0,0001$ & $5,96(2,56-13,89)$ \\
Desempenho escolar abaixo da média & 29,50 & $<0,0001$ & $4,37(2,57-7,43)$ \\
\hline
\end{tabular}

CEBF: cefaleia episódica de baixa frequência ( $<5$ crises $/ \mathrm{mês}$ )

CEFI: cefaleia episódica de frequência intermediária ( 5 a 9 crises $/ \mathrm{mês}$ )

CEAF: cefaleia episódica de alta frequência (10-14 crises/mês)

CCD: cefaleia crônica diária (> 14 crises/mês)

\section{CONCLUSÕES}

Os resultados do presente estudo sugerem que a migrânea e o TDAH são comórbidos na infância, podendo agregar e somar impacto sobre a qualidade de vida, saúde mental e desempenho escolar da criança. Esses dados apresentam implicação imediata na clínica de crianças com cefaleia e na elaboração de programas de saúde pública dirigidos à infância. Diante de uma criança com cefaleia, o clínico deve ampliar sua investigação em busca de sintomas como desatenção, hiperatividade, impulsividade e disfunções executivas, bem como avaliar seu estado de saúde mental e funcio- namento escolar (desempenho, assiduidade, etc.). Ao identificar a comorbidade entre migrânea e TDAH o clínico deve oferecer uma abordagem terapêutica multimodal, dirigida a ambas as condições e baseada nas melhores evidências científicas disponíveis, reduzindo o impacto sobre a vida da criança e maximizando a eficácia do tratamento.

\section{REFERÊNCIAS}

1. Abu-Arafeh I, Razak S, Sivaraman B, Graham C. Prevalence of headache and migraine in children and adolescents: a systematic review of population-based studies. Developmental medicine and child neurology. Dec 2010;52(12): 1088-1097. 
2. Polanczyk G, de Lima MS, Horta BL, Biederman J, Rohde LA. The worldwide prevalence of ADHD: a systematic review and metaregression analysis. The American journal of psychiatry. Jun 2007; 164(6):942-948.

3. Powers SW, Patton SR, Hommel KA, Hershey AD. Quality of life in childhood migraines: clinical impact and comparison to other chronic illnesses. Pediatrics. Jul 2003; 1 12(1 Pt 1):e 1-5.

4. Bellini B, Arruda M, Cescut A, et al. Headache and comorbidity in children and adolescents. J Headache Pain. 2013;14:79.

5. Arruda M, Bigal M. Migraine and migraine subtypes in preadolescent children: association with school performance. Neurology. Oct 30 2012;79(18):1881-1888.

6. Arruda MA, Arruda R, Guidetti V, Bigal ME. Psychosocial adjustment of children with migraine and tension-type headache - a nationwide study. Headache. Feb 2015;55 Suppl 1:39-50.

7. Mattos P, Serra-Pinheiro MA, Rohde LA, Pinto D. Apresentação de uma versão em português para uso no Brasil do instrumento MTA-SNAP-IV de avaliação de sintomas de transtorno do déficit de atenção/hiperatividade e sintomas de transtorno desafiador e de oposição. Rev Psiquiatr Rio Gd Sul. 2006;28 (3):290-297.

8. Fleitlich B, Goodman R. Social factors associated with child mental health problems in Brazil: cross sectional survey. BMJ. Sep 15 $2001 ; 323(7313): 599-600$.

Correspondência
Marco Antônio Arruda
Instituto Glia
Av. Braz Olaia Acosta, 727, s. 310
Ribeirão Preto, SP, Brasil
arruda@institutoglia.com.br

Recebido: 05 de outubro de 2016

Aceito: 10 de dezembro de 2016 